\title{
„Tahrirplatz in Köln"? - Sexualisierte Gewalt im öffentlichen Raum zwischen Aneignung und Zuschreibung
}

\section{Marei Schmoliner}

Nach den Übergriffen auf Frauen* in der Kölner Silvesternacht 2015 war für Polizei und weite Teile der Öffentlichkeit schnell klar, dass es sich um ,,arabisch/nordafrikanische Täter“ (MIK 2016: 1) gehandelt haben musste, ohne dass bereits Klarheit über die Geschehnisse herrschte. Diese Nacht wurde „zur politischen Projektionsfläche [...] bevor sie überhaupt begriffen wurde“ (Amjahid et al. 2016: 18). Im deutschen Mediendiskurs lässt sich beobachten, dass der Sexismus der Anderen herausgestellt und diesen Anderen ein Wir entgegengestellt wird. Dabei verschob sich Gayatri Spivaks (1988: 296) „white men are saving brown women from brown men“ zu einem white men are saving white women from brown men. Nicht mehr am Hindukusch oder andernorts außerhalb Europas sollten Frauen*rechte verteidigt werden, mittlerweile sei der ,öffentliche sexuelle Terror“ ,gegen Frauen [...] aus Nordafrika und Nahost auf Europa übergeschwappt" (Schwarzer 2016: 22; 16). Die Übergriffe wurden als kulturelle Spezifik der Anderen dargestellt. Darauf reduziert waren sie von anderen Erklärungsfaktoren isoliert. Diese Vorgehensweise ist nicht neu, erfahren doch auch andere Gewaltphänomene, die im Rahmen von Fragen der Geschlechterordnung diskutiert werden, gegenwärtig besondere Aufmerksamkeit.

Ricarda Drüeke (2016: 34) fasst für die Berichterstattung von ZDF und ARD zusammen, dass Sexismus und sexualisierte Gewalt „kulturalisiert“ würden und die Berichterstattung ,auf nicht-Weiße [sic] Täter“ fokussiere. Hierdurch stünden „Forderungen nach einer Verschärfung des Asylrechts im 
Mittelpunkt“. Sexismus werde nicht als strukturelles Problem diskutiert, wodurch „die Deutung der strukturellen Zusammenhänge“ nicht möglich sei. Weitergehend werde die „Perspektive der Opfer [...] so gut wie nicht einbezogen." Zusätzlich erfolgt eine Konnotation sexualisierter Gewalt mit Sexualität, was den inhärenten Machtaspekt verschleiert und die Gefahr einer Bagatellisierung und nicht selten auch einer Entkriminalisierung birgt. Das titelgebende Zitat von Alice Schwarzer (2016: 7) wie auch andere (Print-)Medien und der Bericht des Ministeriums für Inneres und Kommunales des Landes Nordrhein-Westfalen (2016: 15) stellen weitergehend einen angenommenen Bezug zwischen der ,gemeinsame[n] sexuelle[n] Belästigung in Menschenmengen [...] anlässlich der ägyptischen Revolution“ und den Ereignissen auf dem Kölner Bahnhofsvorplatz her. ${ }^{1}$ Damit wird sexualisierte Gewalt außerhalb Deutschlands verortet und diese Praktiken werden pauschal arabischen Männern zugeschrieben. Die Übergriffe müssen aber vor einem globalgeschichtlichen Hintergrund in weiteren Aspekten als nur dem des Kulturhintergrunds mit all den inhärent rassistischen Zuschreibungen beleuchtet werden. Von der Annahme ausgehend, dass Ereignisse in der globalisierten Welt untrennbar mit Geschichte und Struktur von Gewalt in Deutschland und in Europa und damit auch mit Kolonialgeschichte verknüpft sind, sollen die Übergriffe in der Silvesternacht hier in einen breiteren sozioökonomischen Kontext eingeordnet werden, als es die öffentliche Debatte, welche der Silvesternacht folgte, vermocht hat. Dabei werden die Ereignisse als im Auftreten oft vergleichbar herausgearbeitet, um sie als gesellschaftliches Phänomen und damit auch als gesamtgesellschaftliches Problem begreifbar zu machen.

Die folgenden Überlegungen wollen die Ereignisse der Silvesternacht als Gewalt gegen Frauen* einordnen und erstreben dabei eine Dekonstruktion der vermeintlich kulturell zuzuordnenden Gruppe der Täter. Sexualisierte Gewalt ist ohne Wenn und Aber abzulehnen und zu bekämpfen. Dabei gilt es trotzdem, die von Chandra Mohanty (1984: 342) geäußerte Einschätzung zu beachten, es sei ,problematical to speak of [...] Arab and Muslim societies (i.e., over twenty different countries) without addressing the particular

1 Eine Analyse der Medienberichterstattung findet sich bei Hark/Villa (2017: 35ff). Die Autor_innen arbeiten weitergehend in einem ganzen Kapitel die ,toxische Aufladung von Schwarzers Feminismus“ (ebd.: 89) durch Kulturessentialismus mit besonderem Fokus auf die Kölner Silvesternacht heraus. 
historical, material, and ideological power structures that construct such images." Anstatt die Ergebnisse generalisierend für die komplette arabische Welt zu proklamieren, diskutiert dieser Beitrag die Ereignisse auf dem Kairoer Tahrirplatz und dem Kölner Bahnhofsvorplatz und strebt dabei an, die Übergriffe in ihrem jeweiligen Kontext als Gewaltphänomene herauszuarbeiten, welche nicht isoliert von ihren Verknüpfungen mit Machtverhältnissen betrachtet werden können. Hierbei soll aufgezeigt werden, dass sexualisierte Gewalt nicht außerhalb ihrer Zusammenhänge verhandelt werden kann und sollte. Es wird dabei weniger darum gehen, was wirklich geschah, sondern ich möchte mich vielmehr auf die Konstruktion des Anderen in Köln und Kairo konzentrieren. Die sexualisierte Gewalt soll dabei im Geflecht der die Gesellschaft durchziehenden Machtstrukturen nachgezeichnet werden, wobei besonderes Augenmerk auf die Herstellung von Männlichkeit durch die Aneignung von Räumen gerichtet wird. Hierfür wird zunächst der öffentliche Raum als Verhandlungsort gesellschaftlicher Macht analysiert, wobei die als gegensätzlich konstruierte Kategorisierung in die private und öffentliche Sphäre im Zentrum steht. Hernach wird mit Raewyn Connells Konzept der hegemonialen Männlichkeit gezeigt, wie die Ereignisse in Köln und Kairo als symbolische Herstellung von Männlichkeit gedeutet und in ein hierarchisches Geschlechtersystem eingeordnet werden kann. Es ist davon auszugehen, dass marginalisierte Männlichkeit über die Einnahme des physischen Raumes ihre Stellung im sozialen Raum zu verbessern sucht. Jedoch sind diese Positionskämpfe mit Connell nur innerhalb komplexer Machtstrukturen zu verstehen. Männer* sind sowohl Täter als auch Opfer von (sexualisierter) Gewalt, wobei sich dieser Beitrag auf die Darstellung heterosozialer Übergriffe konzentriert, die gegen Frauen* gerichtet sind. Männlichkeit wird dabei nicht als statisches Konzept angenommen, sondern als Analysekategorie im Zusammenspiel mit weiteren Faktoren beleuchtet. Hierfür werden die Analogien des Antagonismus in der Herstellung von Geschlecht (doing gender), Räumen, aber auch der Konstruktion des Eigenen und Anderen in beiden Kontexten in ihren wechselseitigen Verflechtungen in den Blick genommen. Untersucht werden sollen die mediale Darstellung sexualisierter Gewalt in der Silvesternacht 2015 in Köln und jene während der Demonstrationen und Feierlichkeiten zum zweiten Jahrestag der Revolution am 
25. Januar 2013 in Kairo. ${ }^{2}$ Grundlage der Analyse bildet der Bericht des Ministeriums für Inneres und Kommunales (MIK) sowie eine vom El-Nadeem Center (2013) zusammengestellte Sammlung von Zeug_innenberichten. Neben der medialen Debatte hat auch der staatliche Sicherheitsapparat versucht, die Ereignisse in Köln aufzuarbeiten, wobei allerdings die bisherigen Ermittlungserkenntnisse weitgehend den bereits am 10. Januar im Ministeriumsbericht genannten Annahmen entsprechen. Im Vergleich fällt auf, dass die Anzeigen aus der Kölner Silvesternacht und die Berichte von Überlebenden der Gewalt auf dem Tahrirplatz auf eine ähnliche Vorgehensweise der Täter, wie auch auf übereinstimmende Täterbeschreibungen verweisen. Die Schauplätze sind zudem Orte des öffentlichen Raumes und die Übergriffe erfolgen aus einer größeren Gruppe heraus (vgl. Schmoliner 2017: 6ff).

Wenn hier von Männern und Frauen die Rede ist, bezieht sich dies auf die gelesene Geschlechtsidentität einer Person, denn die Machtausübung durch sexualisierte Belästigung und Gewalt im öffentlichen Raum basiert häufig auf der nicht vom Individuum, sondern von der Außenwelt vorgenommenen Kategorisierung in männlich und nicht-männlich, wobei alles NichtMännliche als abseits der Norm wahrgenommen wird. In Ägypten und Deutschland wird Geschlecht nicht als fluides Konzept anerkannt, die binäre Zuteilung bleibt hegemonial. ${ }^{3}$ [MATERIALITÄT] Die Sanktionierung alles Nicht-Männlichen schließt LGBTQI*-Personen ein. Trotz Bemühungen um eine pluralisierte Darstellungsweise lassen sich essentialisierende Aussagen

2 Ich spreche hier wie viele Ägypter_innen dezidiert von Revolution und nicht wie viele westliche Medien reduktionistisch vom ,arabischen Frühling“ oder einer „Rebellion“.

3 Diese „Alltagstheorie der Zweigeschlechtlichkeit“ (Hagemann-White 1984) wird als natürliche Voraussetzung sozialen Handelns betrachtet. Sie ist allerdings Resultat einer sozialen Praxis. Mit dem Verweis auf gender wird hier zwar keine Verbindung zwischen einer zweigeschlechtlich deklarierten körperlichen Morphologie und einem geschlechtsspezifisch wahrgenommenem Verhalten angenommen, aber in Gesellschaften, in denen Zweigeschlechtlichkeit die vorherrschende Norm ist, wird ein abweichendes Verhalten möglichst sanktioniert, um das zweigeschlechtliche System aufrecht zu erhalten. Auch wird die gesamte Sozialisationsarbeit darauf ausgerichtet, zwei unterschiedliche Arten von Habitus auszuprägen (vgl. Bourdieu 2005: 43ff). 
nicht komplett vermeiden, will man* die Existenz von Gruppen in ihrer Konstruiertheit aufzeigen. Auf diese Homogenisierungen wird hier bewusst zurückgegriffen, denn ohne sie können die Machtstrukturen im Diskurs nicht benannt werden. ${ }^{4}$

\section{DER „TAHRIR-PLATZ IN KÖLN“ AUS POSTKOLONIALER PERSPEKTIVE}

Die von Schwarzer, dem Ministerium und anderen vorgenommene Übertragung der Ereignisse auf dem Tahrirplatz in Kairo nach Köln ist zunächst einmal unzulässig, denn laut dem BKA befand sich nicht ein einziger Ägypter unter den Tätern (vgl. ZEITonline 2016). Vielmehr dient die Übertragung dazu, sexualisierte Gewalt als der arabischen Kultur inhärent zu kennzeichnen, womit der Sexismus der deutschen Mehrheitsgesellschaft ausgeblendet wird. Das Übertragen verbleibt in der Tradition der Konstruktion einer arabischen Kultur bzw. eines Orients und negiert dabei unterschiedliche Gesetzgebungen, Geschlechterverhältnisse, Sexualitäten und gesellschaftliche Codes in den Ländern der Region. ${ }^{5}$ Werden die Ereignisse von Kairo und Köln jedoch aus einer kontextbezogenen und historisierenden Perspektive analysiert, stellt sich die einfache Übertragung und damit Gleichsetzung der Gewalt von Kairo auf die Gewalt von Köln, trotz der zunächst erkennbaren Gemeinsamkeiten, als Kurzschluss heraus. Eine solche Perspektive bezieht

4 Trotz der Notwendigkeit begrenzender Zuweisungen, sollte die Problematik, hiermit Ausschlüsse und Missrepräsentationen zu schaffen, nicht aus dem Blick geraten. Als Erinnerung werden Bezeichnungen mit dem * versehen, um Spielraum für eigene Geschlechtsdefinitionen und -performances zu geben. Bei (auch indirekten) Zitaten wird auf eine pluralisierte Darstellung verzichtet um hervorzuheben wie Sprache zu Zuschreibungs- und Subjektivierungsprozessen zur Herstellung und Aufrechterhaltung der Geschlechterordnung beiträgt.

5 Diese essentialistischen Zuschreibungen und Stereotypisierungen dienen dazu, die arabische Welt als statisch, homogen und minderwertig zu konstruieren. Dieser Orientalismus nutzt weiterhin machtpolitischen Interessen und der Versicherung des Westens seiner eigenen kulturellen und intellektuellen Überlegenheit. Meyda Yeğenoğlu (1998) zeigt wie diese Unterschiede seit Jahrhunderten über die Diskussion um Geschlechterrollen und Sexualität konstruiert werden. 
bestehende Machtverhältnisse mit ein und folgt damit der Forderung von Reuter und Villa (2010: 17) nach Widerstand gegen ,unilineare, eurozentrische, vermeintlich unpolitische und damit ebenso einseitige wie diskriminierende Mainstream-Theorien von kultureller/nationaler Identität, gesellschaftlichen Machtbeziehungen [...] und deren [...] Diskursivierung." Dabei soll hier aus einer postkolonialen Perspektive betrachtet werden, wie der Tahrirplatz in Kairo und der Bahnhofsvorplatz in Köln in Beziehung gesetzt werden können, ohne orientalistische Stereotype fortzuschreiben und diese zur Konstruktion des Anderen und Eigenen zu nutzen. Postkoloniale Ansätze, mit Bettina Engels (2015: 310) als ,eine politisch motivierte Analysekategorie"verstanden, welche die Kontinuität von Kolonialismus und Dekolonisierung aufzeigt, können vielversprechende Impulse geben. Sie können dabei helfen, eine differenziertere Sichtweise auf die Komplexität struktureller Gewaltverhältnisse zu erlangen. Mit dem Verweis auf die historischen Dimensionen sozialer Ungerechtigkeit sensibilisieren sie gleichzeitig für neokoloniale Herrschaftsformen. Postkolonial ist dabei nicht als eine historisch-räumliche Kategorie zu verstehen, sondern als ein Bezugspunkt der Theoriebildung und empirischen Analyse der machtvollen Wirkung des Kolonialismus bis in die Gegenwart in Politik, Gesellschaft und Wissenschaft in den ehemalig kolonialisierten Ländern, wie auch in den Staaten der ehemaligen Kolonialmächte (vgl. Aikins/Bendix 2010: 479f). Auch ist festzuhalten, dass „der Westen“ und „der Süden“ keine homogenen Kategorien sind. Viele der Beobachtungen über die Gesellschaften des Südens sind relevant für manche Staaten im Globalen Norden, während sie auf andere Staaten im Süden nicht zutreffen mögen.

Wie kann also eine Definition von sexualisierter Gewalt aussehen, die alle Dimensionen und Praktiken von Gewalt gegen Frauen* umfasst und zugleich Freiheits- und Handlungspotenzial für Frauen* ermöglicht, ohne zu einer Viktimisierung von Migrant_innen oder Tätern* zu führen? Hier wird mit einem intersektionalen, kontextbezogenen und diskursiven Verständnis von (sexualisierter) Gewalt gearbeitet (vgl. z.B. Sokoloff/Dupont 2005), um die Gewalt in Köln und auf dem Tahrirplatz an der Schnittstelle von race, gender und anderen Kategorien zu begreifen. Unabhängig von den Diskursen um globale Schwesternschaft und der Dekolonisation von Wissenschaft (vgl. z.B. Marx Feree/Tripp 2006; Mohanty 2003) plädiere ich demnach dafür, die Selbstbestimmung über ihren Körper als Ziel und Recht von Frauen* überall 
auf der Welt anzunehmen. Mögen die Diskurse und Ereignisse sich auch unterscheiden, die Forderung nach einem Ende sexualisierter Gewalt wurde sowohl in Kairo als auch in Köln geäußert. Die postkoloniale Ebene soll in dieser Arbeit helfen, die binären Folien, die in den deutschen Quellen verwendet werden, aufzuzeigen, während gleichzeitig der postkolonial-feministischen Forderung nachgekommen wird, symbolische oder epistemische Gewalt nicht getrennt von institutionalisierten Machtverhältnissen zu analysieren (vgl. z. B. Castro Varela/Dhawan 2005: 8). Gerade die Perspektive auf materielle und politische Implikationen eröffnet einen solchen Blick auf die angesprochenen Phänomene und ihre Deutungen, wie ich nun an raumtheoretischen Erwägungen genauer zeigen werde.

\section{RÄUME ALS VERHANDLUNGSORTE GESELLSCHAFTLICHER MACHT}

Der öffentliche Raum ist niemals nur Raum im physischen Sinne, sondern immer auch mit Sinn gefüllt und durch Praktiken hergestellt. Er muss deshalb im Rahmen mindestens zweier Deutungsmuster untersucht werden: im Sinne des materiellen Raumes (public space) wie auch des metaphorischen Raumes (public sphere). Für eine Untersuchung des Faktors Raum in den Ereignissen von Kairo und Köln ist es zunächst notwendig, Raum als Verhandlungsort gesellschaftlicher Macht zu begreifen. Ebenso wie die Konstruktion von (Geschlechts-)Identität basieren auch Raumstrukturen auf dem Differenzprinzip. So wie Männlichkeit häufig in Abgrenzung zu Weiblichkeit hergestellt wird und vice versa, werden Räume über die binäre Einteilung in öffentlich/privat oder gefährlich/sicher konstruiert. Sie spiegeln also gesellschaftliche Organisationsprinzipien und Machtverteilungen wider. Die Legitimierung von Inklusion und Exklusion stehen im öffentlichen Raum somit unaufhörlich zur Diskussion und dies spielt beim Zusammenwirken von Raum und Geschlecht eine besondere Rolle. Mit Sybille Bauriedl und Kolleg_innen (2010: 10) kann davon ausgegangen werden, dass die Weise, in der öffentliche Plätze genutzt werden, ,,weder zufällig noch geschlechterbiologisch bedingt noch stadtphysiologisch vorgegeben, sondern gesellschaftlich hergestellt“" ist. Die unsere Alltagswelt strukturierenden Machtverhältnisse manifestieren sich somit auch in verräumlichten Geschlechterverhältnissen, welche dazu beitragen, Machtstrukturen und -asymmetrien zu verfestigen und aufrecht zu 
erhalten. Eine Untersuchung von Raum ermöglicht es, als gegeben angenommene räumliche Verhältnisse als Resultat menschlichen Handelns und die damit herbeigeführten Strukturierungen physisch-materieller Bedingungen ins Blickfeld zu nehmen.

Anouk de Koning (2009: 141) zeigt für öffentliche Orte in Kairo, wie Räume geschlechtlich konnotiert sind. In ihrer Studie wird offenbar, wie schon die bloße Anwesenheit einer Frau* an einem bestimmten Ort und zu einer bestimmten Zeit in Kairo als Indikator für ihren Anstand genommen wird und wechselseitig Orte durch geschlechtsspezifische Zugänglichkeit konstruiert werden. Weiterhin zeigt sie, wie auch Klasse in Kairo als Marker genutzt wird, um Zugang zu Räumen zu beschränken, das Andere zu konstruieren und somit der öffentliche Raum für Aushandlungsprozesse genutzt wird (vgl. ebd.: 152f). Für den deutschen Kontext haben Karin Kutschinske und Verena Meier (2000) potentielle Angsträume von Joggerinnen als Ausdruck von Geschlechterkonstruktionen sichtbar gemacht und dabei dargelegt, dass weder die Bedeutung von Angsträumen noch die (weibliche*) Erfahrung von Angst vor sexualisierter Gewalt im öffentlichen Raum dem Raum oder Frauen* inhärent sind, sondern gesellschaftlich konstruiert. Dabei hat die in Einzelfällen ausgeübte Gewalt symbolische Auswirkung auf alle Frauen* und führt so zu Einschränkungen der Bewegungsfreiheit in zeitlicher und räumlicher Hinsicht. Diese rigiden Normierungen des Raumes haben Einfluss auf den gesamten Lebensentwurf von Frauen*, auch weil in für sie unzugänglichen Räumen ausgeübte Gewalt als Bestrafung für abweichendes Verhalten gelesen wird.

Zum einen erlangen Räume also in ihrer Nutzung durch verschiedene Personengruppen verschiedene eingeschriebene Bedeutungen, zum anderen sind die Bedeutungen von Räumen wichtiger Bestanteil im Prozess von Identitätskonstruktion und damit der Reproduktion gesellschaftliche Strukturprinzipien. Dadurch „co-konstituieren“ (Strüver 2003: 114) sich die Bedeutungen von Raum und Geschlecht. Martina Löw (2007: 63f) schlägt mit anderen in Anlehnung an Anthony Giddens eine „Dualität von Raum“ vor. Raum solle als ,relationale (An)Ordnung von Lebewesen und sozialen Gütern an Orten" begriffen werden. Der Begriff der (An)Ordnung zeigt dabei auf, dass Räume auf einer Praxis des Anordnens als wahrnehmend-synthetisierendes Verknüpfen basieren, dass sie aber auch eine gesellschaftliche Ordnung vorgeben. Somit sind Raumordnungen als gesellschaftsstrukturierende Elemente sowohl Bedingung als auch Folge von Handeln. In dieses Muster 
fügt sich auch die „,im historischen Prozess herausgebildete Dichotomie“von öffentlich/privat ein, welche als ,wirkmächtige, ideal-typisch-normative und insbesondere räumlich manifestierte Konstruktion“ (Ruhne 2003: 93) für diese Untersuchung von Bedeutung ist. Einerseits sind diese Kategorien sozial konstruiert, andererseits beeinflussen sie als Materialisierungen gesellschaftlicher Machtverhältnisse, die prozesshaft hergestellt werden, die Herstellung dieser Kategorien selbst.

Das Konstrukt der öffentlichen Sphäre, verstanden als Kernstück der symbolischen Geschlechterordnung wird hier als Schauplatz von Verteilungskämpfen bei der (Re-)Produktion von Machtverhältnissen genutzt. Jedoch bietet diese Einteilung - von Feminist_innen dafür herangezogen, den Ausschluss von Frauen* aus einzelnen Domänen zu kritisieren - in globaler Perspektive Probleme, wenn sie kritiklos für alle Kontexte angewandt wird. Die Einteilung verleitet zur Annahme, dass Frauen* - als zur privaten Sphäre gehörig - machtlos seien, da Männer ein Monopol auf die öffentlich-politische Sphäre hätten. Auch zeichnen sich im deutschen Zusammenhang bezüglich der Zuweisung der Sphären gegenwärtig folgenreiche Transformationen ab, die durch das Eintreten der ersten Welle der feministischen Bewegung für gleichberechtigte Teilhabe von Frauen* in öffentlichen Bereichen wie Bildung, Wissenschaft, Arbeitsmarkt und Politik ermöglicht wurde. Das Erarbeiten von Präsenz auf der Ebene der öffentlichen Meinung in der politischen Sphäre war für das erfolgreiche Wirken der feministischen Bewegung unabdingbar (vgl. Klaus/Drüeke 2010: 251).

Fowziyah Abu-Khalid (2003: 50) proklamiert, dass die arabische Frauen*bewegung noch keinen ,kulturell relevanten Diskurs um die private Sphäre" gefunden hätte und die Kernfrage hier sei, Frauen* innerhalb dieser zu stärken und nicht gegen sie. ${ }^{6}$ Judith Tucker (1985: 102ff) und Margot Badran (1995: 14f) hingegen zeigen im Hinblick auf die Dichotomie der öffentlichen und privaten Sphäre sowohl für urbane als auch rurale Bereiche, dass ägyptische Frauen* an fast allen sozialen und ökonomischen Institutio-

6 Diese Forderung ist im Zusammenhang der Ablehnung hegemonialer Feminismen zu verstehen. Manche Aktivist_innen der arabischen Frauen*bewegung grenzen sich bewusst von ,westlichen“ Emanzipationsformen ab. Die Motivation dafür reicht vom Fokus auf eine breitere Leser_innenschaft bis zur Einschätzung, „westliche“ Feminismen seien nicht auf die eigenen Kontexte anwendbar. 
nen beteiligt waren. Hier sollte allerdings nicht vergessen werden, dass ägyptische Feminist_innen in der Vergangenheit gegen doppelt verankerte patriarchale Strukturen kämpften: die ägyptische und die britische im Rahmen der Kolonisierung. ${ }^{7}$ Ägyptische Feminist_innen haben nach der Unabhängigkeit den vom Staat angebotenen Zugang zur politischen Sphäre zurückgewiesen, da dieser zur Bedingung hatte, sich politisch zu mäßigen und bürgerliche Normen zu befolgen (vgl. Hatem 1994; Bier 2011). ${ }^{8}$ Die Auswirkungen zeigen sich bis heute in mangelnder politischer Repräsentation von Frauen* (vgl. Sorbera 2014: 65). Statt Partizipation zu ermöglichen, wurde für einen erweiterten Politikbegriff plädiert, der jede Form der Aktivität politisch fundiert. Damit wird privates Handeln zur Grundlage öffentlichen Handelns und es wird möglich, auf die Macht von Frauen* in der privaten Sphäre zu verweisen. Frauen* wird dabei zugeschrieben, Männer* in der privaten Sphäre derart zu beeinflussen, dass sie in der öffentlichen Sphäre quasi keine eigenen Entscheidungen mehr treffen. Darüber hinaus sind Frauen* Teil von Netzwerken, welche für die ägyptische Politik zentral sind. Dadurch können sie trotz des Ausschlusses von formaler Partizipation an Entscheidungen teilhaben (vgl. Harders 2009: 303). Dieses Vorgehen lässt sich mit Deniz Kandiyoti (1988) als „Bargaining with Patriarchy“ beschreiben. Frauen* treffen Entscheidungen, die zunächst marginalisierend wirken, gleichzeitig aber Potential für begrenzte Zugewinne, in diesem Fall Partizipation, bieten.

Bei aller Kritik an diesem mangelnden Zugang von Frauen* zur politischen Sphäre darf nicht aus dem Blick geraten, dass ägyptische Frauen* zusätzlich zu den allgemeinen Beschränkungen hinsichtlich politischer Partizipation autoritärer Systeme, denen auch Männer* unterworfen sind, durch patriarchale Strukturen ausgebremst wurden und werden. Die Forderung nach Zugang zur öffentlichen (im Sinne von politisch-diskursiven) Sphäre mag historisch keine dezidierte Forderung ägyptischer Frauen* gewesen sein. Während der Revolution allerdings fanden viele Frauen* durch Schreiben, Aktivismus und die „diskursive Transformation“ (McLarney 2015: 2)

7 Für einen Überblick über die britische Kolonialpolitik in Ägypten mit Bezug auf Geschlechterpolitik und die Konstruktion des Anderen - siehe Braun/Mathes (2007: 309ff).

8 Ich spreche hier dezidiert nicht von bürger_innenlich, sondern nutze das generische Maskulinum um die implizite männliche, weiße, mittelständische Konnotation sichtbar hervorzuheben. 
sozialer, kultureller und politischer Institutionen Zugang zur öffentlichen Sphäre. Um das Konzept des Öffentlichen und Privaten für beide Fälle nutzbar zu machen, kann mit Suad Joseph dafür plädiert werden, mehr Alltagsbezug in die Konzepte zu bringen und sie für alle Kontexte als fluide anzunehmen:

,[T] he categories of public/private $[\ldots]$ - important abstractions in political theory and law - do not translate directly to the lived experiences of women or men in the 'West' or 'South'. The public/private are not as separate in reality in the West as Western classical liberal discourse would imagine." (Joseph 1997: 75)

Über Raumnutzung, -aneignung und -konstitutionen verhandeln Individuen und Gruppen also auch Macht- und Herrschaftsverhältnisse. In Anknüpfung an David Harvey (1989: 233), der behauptet die Ausweitung von Macht beruhe wesentlich auf der Fähigkeit, die Produktion von Raum zu beeinflussen, soll hier die Aneignung von Raum an der Schnittstelle von Machtstrukturen analysiert werden. Analog zum Konzept des doing gender, das Geschlechtszugehörigkeit und -identität als fortlaufendend hergestellt und damit jeder menschlichen Aktivität inhärent begreift, schlage ich ein doing space als Teil dieses Herstellungsprozesses vor. Die Untersuchung geschlechtsspezifischer Mobilitätsmuster ist hinreichend erforscht, wobei hier zumeist auf Sozialisation abgehoben wird, anstatt die unterschiedlichen Raumaneignungsprozesse als aktives Herstellen von Geschlecht zu begreifen. Raumaneignung explizit als Mittel der (Re-)produktion von Geschlecht zu untersuchen ist in vielen Bereichen noch nicht verbreitet. Ein doing im Zusammenspiel der beiden Begriffe, wie im Titel des vorliegenden Sammelbandes, betont den sozialen Prozesscharakter der Notwendigkeit, Raum und Geschlecht beständig zu machen, also herzustellen. [EINLEITUNG] Raum ist, wie gezeigt wurde, geschlechtlich konnotiert. Die Folge davon ist, dass Frauen* und Männer* ihre Geschlechtlichkeit im öffentlichen Raum verschieden darstellen, beziehungsweise über ihre Performanz im öffentlichen Raum Geschlecht erst herstellen. Wie doing space konstitutiv für doing gender ist, zeigt für die ägyptische Gesellschaft Farha Ghannam (2002: 100):

„As men walk, they scan the area around them [...]. Their masculinity and reputation are contingent upon their ability to interfere to protect a woman, correct a misbehavior, or help in rescuing people in an accident. [...] In contrast, a young woman [...] 
should show modesty by taking a serious posture that is manifested in walking fast and avoiding looking at men or responding to their comments."

In diesem Zitat finden sich geschlechtsspezifische Rollenerwartungen der Performanz im öffentlichen Raum (siehe auch Koning 2009: 147). Während Männer sich den öffentlichen Raum aneignen dürfen und sollen, halten Frauen* sich dort nicht grundlos auf. Sie sollen den öffentlichen Raum schnellstmöglich wieder verlassen und durch klare Körperhaltung Anstand demonstrieren. Auch in Deutschland wird von Frauen* und Männern* verschiedene Performanz im öffentlichen Raum erwartet, und es existieren Vorstellungen davon, welche Personen an bestimmte Orte ,gehören“ und welche nicht (vgl. Strüver 2003: 120). Raumaneignung kann also als Teil des doing gender gesehen werden, während gleichzeitig, wie oben ausgeführt, durch gegenderte Körper Raum hergestellt wird. [KÖRPER]

\section{DOING SPACE, DOING GENDER: RAUMANEIGNUNG UND GESCHLECHT IN KAIRO UND KÖLN}

Im Kontext der Revolution auf dem Tahrirplatz kann mit Derek Gregory (2013) von „Performances of Space“ gesprochen werden. Der Platz wird hier zu einer Art Bühne, auf der die Demonstrant_innen agieren. Dabei erfährt der Raum durch die Performanz der Menschen eine Rekonstruktion, die Materialität des Raumes und die Körperlichkeit der Besetzer_innen tragen in ihrer Kombination zu einer Neudefinition des Platzes bei. Die Proteste auf dem Tahrirplatz können als ein reclaim the space gelesen werden, denn nach Hussam H. Salama (2013: 128) wurde der öffentliche Raum von den meisten Ägypter_innen mit „,der Regierung gehörendem Raum“ gleichgesetzt. Die Masse der Demonstrierenden eignete sich den Platz an, welcher der Bevölkerung wegen seiner Bedeutung als Befreiungsplatz gezielt versagt und zu einem Verkehrsknotenpunkt umgestaltet wurde. ${ }^{9}$ Wie dabei auch Geschlechterrollen ,co-konstituiert“ wurden, zeigt sich laut Dina Wahba (2012) durch

9 Der Kairoer Tahrirplatz ist mit historischen Traditionen und Ereignissen verbunden. Seit der Unabhängigkeit steht er für politische Freiheit. Hier feierte König Farouk den Abzug der britischen Truppen 1949 und seitdem war der Platz ein Magnet für öffentliche Aktionen (vgl. Filiu 2011: 61). Tahrir wurde hernach 
die große Zahl von Frauen*, die im öffentlichen Raum demonstrierten und schliefen. Judith Butler (2016: 121) sieht dieses Schlafen als einen politischen Akt, da es ,eine konventionelle Unterscheidung zwischen öffentlich und privat" überwand. Während Butler hier auf die Unterscheidung in politisch und unpolitisch abzielt, es durch das Leben auf dem Platz zu einer Auflösung der Sphären kommt und somit das Private zum Politischen wird, kann es vor dem Hintergrund der Feminisierung der öffentlichen Sphäre auch als ein politischer Akt der Frauen* eingeordnet werden: Indem sie durch ihre Körper im öffentlichen Raum Anspruch auf die Öffentlichkeit proklamierten, stellten sie Geschlechternormen und Machtverhältnisse in Frage. Mit ihrer nächtlichen Präsenz auf dem Platz und dem Schlafen an einem öffentlichen Ort wurden durch alle Demonstrant_innen, insbesondere durch die weiblichen*, Normen gebrochen. [EXPOSURE] Über Körper im Raum wurden also Diskurse um politische Herrschaft und Dominanz ausgetragen, welche auch um den Frauen*körper kreisten. Diese Körper können dadurch als nicht passiv, sondern als Orte, die zwar durch „Patriarchat, Islamismus und säkular-moderne Männlichkeit“ (Hafez 2014: 175) kontrolliert und reguliert werden, gleichzeitig aber auch Orte des Widerstands und Gegendiskurses formten, gesehen werden. Somit wurde mit der Performanz ihrer Körper im öffentlichen Raum, durch ihre schiere Präsenz neue Bedeutungen und Verständnisse eines weiblichen* Körpers im öffentlichen Raum eingeschrieben und der Raum revolutionär geformt. [KÖRPER]

Diese neue Performanz wurde laut Dina Wahba (2012) zunächst akzeptiert: „These were the only 18 days in Egypt in my life that I was never harassed at all." ${ }^{10}$ Während der ersten Tage der Proteste auf dem Tahrirplatz war sexualisierte Belästigung noch selten (vgl. Sobera 2014: 68). Wahba (2012) nennt diese Abwesenheit ein „Indiz für einen gewissen Grad an Inklusion“ und spricht von einer „Aufhebung von Geschlechterrollen“, und dass diese dann ,sehr abrupt“ wiederhergestellt worden seien. Im Kontext der obigen Ausführungen zu doing gender wird hingegen deutlich, dass Geschlechterrollen niemals aufgehoben, sondern nur im transformativen Sinne verändert

umgestaltet, „, for the traffic of vehicles not ideas, and regulated by the security apparatus not the civic body“ (Gregory 2013: 241). Der Platz erlangte 2011 als eröffneter Raum eine zentrale Bedeutung für die Proteste.

10 Wahba spricht hier von den 18 Tagen vom Beginn der Revolution am 25. Januar 2011 bis zum Sturz Muaraks am 11. Februar. 
werden können. Mit Butler (1997: 32f) wird dann die „sedimentierte Wirkung einer andauernd wiederholenden oder rituellen Praxis“, durch die Geschlecht hergestellt wird, unterbrochen, wodurch die Normen, welche das doing gender konsolidieren, in eine ,produktive Krise“ versetzt werden. In diesem Zusammenhang wäre auch die Transformation von Geschlechterrollen für einen gewissen Zeitraum möglich, in dem sie subversiven Widerstand zuließen. Durch das Einkreisen von Frauen* und massive sexualisierte Gewalt ab Winter 2012 wurde die (patriarchale) Geschlechterordnung wiederhergestellt, wie auch Whaba (2012) berichtet. Hernach stiegen die Fallzahlen rasant (vgl. Hafez 2014: 183; Abdelmonem 2016: i). Mit Butler (1988: 522) kann der Einsatz und Anstieg sexualisierter Gewalt als Bestrafung für Abweichung gedeutet werden. Frauen* wichen als politischer Akt von gängigen Vorstellungen von Geschlechterrollenbildern ab und versagten damit in der Performanz ihres Geschlechts. Damit stellten sie nicht nur das staatliche System in Frage, sondern auch die gesellschaftlich vorgesehene zweigeschlechtliche Ordnung, die Frauen* einen Platz außerhalb der öffentlichen Sphäre zuschreibt.

Es zeigt sich also, welch enormes Transformationspotential in der Performanz des öffentlichen Raumes durch Frauen* liegt. Die späteren Angriffe zielten darauf ab, Frauen* durch Verdrängung aus der öffentlichen Sphäre als politische Subjekte anzugreifen. Durch Belästigung und Gewalt sollte sichergestellt werden, dass sie ihre Staatsbürger_innenrechte nicht mehr wahrnehmen können. Macht, Raum und Geschlecht verweben sich also und führen zum Ausschluss von ägyptischen Frauen* aus der öffentlichen Sphäre. Damit sollte ihnen, aber auch der Revolution geschadet werden, die damit sichtbar einen Teil ihrer Unterstützer_innen verlor. Diese Prozesse des Aneignens des Raumes durch Frauen* und der Reaktion der Verdrängung aus dem öffentlichen Raum durch sexualisierte Gewalt, um Frauen* wieder auf ihren Platz zu verweisen, bestätigen die obige These, auf dem Tahrirplatz wären Machtverhältnisse im öffentlichen Raum ausgehandelt worden. Dabei zeigt sich, dass hier sowohl Prozesse konkreter Aneignung ablaufen, als auch die Demonstration von Macht durch die erneute Verdrängung aus der öffentlichen Sphäre. Da, wie Maria Spitthöver (1990: 81) anmerkt, „Macht haben und Raum haben [...] in der Regel in engem Zusammenhang stehen“, kann die Aneignung des Raumes auch einen Zugewinn von Macht bedeuten. Empfundene Machtlosigkeit kann dann bei der Aneignung des Raumes eine Rolle spielen. 
In den Erziehungs- und Sozialwissenschaften werden die Zusammenhänge zwischen Anerkennung, Macht und Gewalthandeln seit den 1990er Jahren diskutiert. Dabei wurde gezeigt, wie Jugendgewalt aus vorhergehenden Erfahrungen von Missachtung, also mangelnder Anerkennung entsteht und damit als ,dynamischer Folgezusammenhang“ verstanden werden muss (vgl. z.B. Thompson 2015: 55). Hierbei spielt nicht nur interpersonell erlebte Gewalt (in Form von Missachtung) eine Rolle, sondern auch symbolische Gewalt. Individualtheoretische Perspektiven, welche Subjekten erst die Möglichkeit geben als Subjekte gesehen zu werden, bringen Diskriminierungs- und Ausgrenzungserfahrungen ans Licht.

Viele der Täter von Silvester in Köln stammen aus einem ,sehr spezifische[n] Milieu“ (Amjahid et al. 2016: 27), das sich aus Menschen aus Marokko und Algerien, welche keine langfristige Bleibeperspektive in Deutschland haben, zusammensetzt. ${ }^{11}$ Sie befinden sich als Asylsuchende oder ,klandestin Migrierende“ (Zillinger 2016: 52) in Abhängigkeit von der Qualität ihres Aufenthaltsstatus, womit verschiedene gesetzlichen Restriktionen, soziale und institutionelle Diskriminierungen, aber auch Alltagsrassismus und damit eine äußerst prekäre Lebenslage in Deutschland verbunden sind. Der (angenommene) Bildungsstand dient im Kontext von Migration in problematischer Weise dazu, im kapitalistischen System zwischen „brauchbaren“ und ,unbrauchbaren“ Individuen zu unterscheiden und die Anderen von der Mehrheitsgesellschaft abzugrenzen. Wer sich dadurch als „Verlierer der Wettbewerbsgesellschaft“ sieht und „keine klaren Entfaltungsperspektiven für [seine] soziale und berufliche Zukunft" sieht, neigt laut Heidrun Bründel und Klaus Hurrelmann (1994) zu Aggression und Gewalt im Jugendalter. Mit den Worten von Youssef, eines Täters von Silvester: „Ich habe in meinem ganzen Leben nichts erreicht. [...] Ich bin ein Verlierer" (Amjahid et al. 2016: 22). Die Kölner Täter haben, während ihre Verfahren laufen, keine Möglichkeit zu arbeiten und damit in einer Gesellschaft, die so erwerbszentriert ist wie die deutsche, kaum Möglichkeiten, sozial aufzusteigen. Daraus erwächst ihre Situation, keine sinnstiftende Aufgabe zu haben, woraus wiederum das Gefühl resultiert, „vor Langeweile“ (ebd.: 34) zu sterben.

11 Eine genauere Betrachtung dieser Milieus bietet Martin Zillinger (2016). Er zeigt wie „klandestin Migrierende“ manchmal bis zu zehn Jahre in der Illegalität leben, bis sie Papiere erhalten. Diese Jahre zeichneten sich durch Entbehrungen und eine Entfremdung von ihren Familien aus. 
Diskriminierungserfahrungen ziehen also Aneignungs-, Umdeutungsund Abwehrstrategien nach sich, die sich auch gewaltvoll ausdrücken können. Die Jugend der Täter in Kombination mit Migrations- und Marginalisierungsprozessen kann eine Reaktion wie die in Köln hervorrufen. Darauf deutet auch die Forschung von Paul Silverstein hin, der beschreibt, wie junge franko-arabische Männer in der Pariser Banlieue auf die Kriminalisierung, racial profiling und das konstante Überwachen und Drangsalieren durch öffentliche und private Sicherheitskräfte mit einer Aneignung des Raumes durch die gewaltvolle Demonstration von Männlichkeit in der Metro reagieren. Hier wird weiter klar, wie ,europäische Ängste‘ zur Kontrolle ,arabischer Körper' im öffentlichen Raum durch Segregation, Legislation und spezielle Verhaltensnormen zu einer veränderten Raumwahrnehmung frankoarabischer Jugendlicher führen. Silverstein (2008: 171) macht damit die Verknüpfung von race und Raum deutlich und hebt dabei (männliche) Gewalt als eines der strukturierenden Elemente hervor (vgl. für Deutschland Schmincke 2009). [KÖRPER]

Wenn mit Markus Schroer (2007: 258) weiterführend davon ausgegangen wird, dass es für Individuen bedeutsam ist „wahrgenommen und damit sichtbar" zu sein und Gewalt dabei ein ,bevorzugtes Mittel darstellt, um auf sich aufmerksam zu machen" (ebd.: 267), wird das Zünden von Feuerwerkskörpern, ,wobei diese auch gezielt auf Personengruppen und auch Einsatzkräfte der Polizei gefeuert wurden“ (MIK: 1) als Raumaneignung und somit als visuelles Symbol im Kontext des doing space offenbar. Diese Aneignung als Gegenstrategie zu Marginalisierung ist mit männlichem Dominanzgehabe und Körperlichkeit verknüpft. Bedenkt man, dass der Ausschluss von Frauen* bereits ein „konstitutives Moment des öffentlichen Raumes“(Ruhne 2003: 98) ist, so wird klar, dass die Ereignisse der Kölner Silvesternacht als ein doing space des gewaltvollen Aneignens des öffentlichen Raumes durch ein Aneignen von Frauen*körpern gelesen werden können.

Vor dem Hintergrund dieser Ausführungen werden zwei verschiedene Muster offenbar: Wurde in Kairo sexualisierte Gewalt auf Frauen*körper angewendet, um Frauen* als politische Subjekte aus dem öffentlichen Raum als politischer Sphäre auszuschließen, wurde in Köln die Gewalt benutzt, um auf sich aufmerksam zu machen. Anstatt eines Ausschlusses, ging es darum, die eigene Ausgeschlossenheit sichtbar zu machen, welche mit dem Status einer migrierten Person verknüpft ist. [EXPOSURE] [EINLEITUNG] Die 
von Bourdieu (1991: 26) geäußerte Einschätzung, dass „,der von einem Akteur eingenommene Ort und sein Platz im angeeigneten physischen Raum hervorragende Indikatoren für seine Stellung im sozialen Raum abgeben“, kann nach Obigem dahingehend interpretiert werden, dass marginalisierte Männlichkeit über die Einnahme des physischen Raumes ihre Stellung im sozialen Raum zu verbessern sucht. „Der angeeignete Raum ist einer der Orte, an denen Macht sich bestätigt und vollzieht““ (ebd.: 27).

\section{GEWALT ALS DOING SPACE ZUR HERSTELLUNG VON MÄNNLICHKEIT UND PATRIARCHAT}

Wie ich eingangs hervorgehoben habe und an anderer Stelle ausführlicher zeigen konnte, hängen die Konzepte des doing gender und des doing space eng zusammen. Der männliche Übergriff auf den weiblichen* Körper war an beiden Orten Ausdruck des Aushandelns von gesellschaftlicher Macht (vgl. Schmoliner 2017). Der weiblichen Opfergruppe wird im deutschen Mediendiskurs eine männliche Tätergruppe gegenübergestellt. Auch das Ministerium (2016: 1) geht von einer homogen weiblichen Gruppe Betroffener aus: „Opfer waren nahezu ausschließlich Frauen“. Für die ägyptischen Zeug_innenberichte gilt dasselbe. Hier wird ebenfalls herausgestellt, dass nur Frauen von sexualisierter Gewalt betroffen waren (vgl. El-Nadeem 2013: 28f, 32f, 38-43).

Das feministische Patriarchatskonzept, welches genderbasierte Diskriminierungen als Teile eines übergreifenden Phänomens männlicher Dominanz begreift, konnotiert Männlichkeit generalisierend mit Macht. Dies erschwert die Berücksichtigung der Unterschiede in männlichen* Lebenslagen, in denen Männer* sich sowohl in Macht-, als auch in Ohnmachtspositionen wiederfinden können. Eine Untersuchung von Machtbeziehungen bedarf deshalb der Erweiterung um andere Kriterien, um das Phänomen erfassen zu können, wie es Raewyn Connell (2015 [1987]) mit ihrem Konzept der hegemonialen Männlichkeit, die als Ideal gesetzt wird, nach dem alle Männer* streben, getan hat. Ohne diese Dimension der Geschlechterordnung kann männliche* Gewalt nur unzureichend erfasst werden, da die Gefahr besteht, in der Binarität zwischen männlich-weiblich und damit Täter-Überlebende zu verbleiben, ohne die Zwischenräume in Betracht zu ziehen. Denn „Masculinity is not $[. .$.$] the experience of power; it is the experience of entitlement$ 
to power" (Kimmel 2007: 101, Hervorhebung im Original). Michael Kimmel (ebd.: 102) hat weitergehend herausgearbeitet, wie ,[a]gain and again, [...] men initiate violence when they feel a loss of power to which they felt entitled." Weitergehend muss einer Sichtweise Gewaltbetroffener als passive Opfer entgegengewirkt werden, um die Handlungsmacht von Frauen* nicht auszublenden.

Auch in arabischen Ländern wird Männlichkeit mit Macht assoziiert. Gleichzeitig ist das verlangte Ernährermodell von jungen Männern durch die hohen Arbeitslosenzahlen nicht mehr erreichbar (vgl. El-Feki et al. 2017: 25). Männlichkeit kann also auf diese Weise nicht mehr hergestellt werden. Zusätzlich tritt in Ägypten die männliche Erziehung zur kontrollierenden Person mit der Unterdrückung durch staatliche Institutionen in Konflikt (vgl. Hafez 2012: 39). Wenn gesellschaftlich prekär gewordene Männlichkeit bedroht ist, setzen Männer häufig Gewalt als letzte Ressource ein, um Männlichkeit zu performen (vgl. Messerschmidt 1993: 5). Die gewaltvolle Bestätigung ihrer Maskulinität ist dann - gerade für marginalisierte Jugendliche die einzige Möglichkeit sich als männlich zu positionieren. Männlichkeit und die damit einhergehenden Privilegien werden unter Zuhilfenahme überzogener Praktiken der Verkörperung von Hypermaskulinität oder aber den Übergriff auf den Frauen*körper wiederherzustellen versucht. Männlichkeit wird gegen die Abwertungen im Kontext von race und Klasse und die marginalisierende Lebenssituation verteidigt. Die strukturelle Ohnmacht kann so durch Machtausübung im öffentlichen Raum kompensiert werden.

Zusammenfassend lässt sich Folgendes festhalten: Gewalt im Geschlechterverhältnis lässt sich als ein Mittel zur Herstellung von situativer oder temporärer Herrschaft begreifen, die, wie gezeigt wurde, ein konstituierendes Merkmal von Männlichkeit darstellt. Wird diese Männlichkeit bedroht oder lässt sich nicht mehr auf die bewährte Art herstellen, greifen Individuen auf Gewalt als letzte Ressource zurück. Das doing space von Köln und Kairo ist damit gleichzeitig auch ein doing gender. Die Reproduktion des patriarchalen Geschlechterverhältnisses über räumliche und körperliche Aneignung dient der Aufrechterhaltung und dem Zugewinn von Macht. Heterosoziale Gewalt dient dann der Herstellung und Reproduktion sozialer Ordnungsmuster, hat aber auch identitätskonstruierende Elemente, wobei auch homosoziale Gewalt als männliches* Gewalthandeln ordnungs(re)produzierend ist (s. Meuser 2002). 
Anknüpfend an Connells Konzept der protestierenden Männlichkeit auf individueller Ebene können die Ereignisse in Köln und Kairo in den Kontext der Wiederherstellung patriarchaler Strukturen gestellt werden. Denn „women's inferior social status is maintained by the state and other institutions and by violence, including sexual violence." (Wood 2006: 325) In Konflikten, so auch während der Revolution auf dem Tahrirplatz, erhalten (staatliche) Institutionen Geschlechterverhältnisse nur noch unzureichend aufrecht und diese Leerstelle wird durch männliche Gewalt zur Aufrechterhaltung der patriarchalen Ordnung gefüllt. Davon ausgehend, dass hierarchisch-patriarchale Geschlechterordnungen für die Reproduktion von Herrschaftsstrukturen und damit in autoritären Regimen zur Aufrechterhaltung der Stabilität nötig sind (vgl. Schneider/Wilde 2012: 9), erscheint diese Lesart für das ägyptische Beispiel schlüssig. Mit Anthony Giddens' (1988: 70) Theorie der Strukturierung können die Übergriffe im Raum im Kontext von „Regeln und Ressourcen, die in die Produktion und Reproduktion sozialen Handelns einbezogen sind, [die] gleichzeitig die Mittel der Systemproduktion darstellen“ verstanden werden. Dann wird der Mechanismus sichtbar, das patriarchale System wie auch das politisch autoritäre Regime wieder herzustellen und zwar über Aneignung des Raumes durch die Aneignung von Frauen*körpern. Damit schließe ich an Susanne Krasmann und Jürgen Martschukat (2007: 10f) an, nach denen ,exzessive Gewalt sich in eine spezifische Rationalität der Machtausübung einfügt, wie der Exzess gewissermaßen Teil eines Programms der Ordnungsherstellung wird.“

Zeug_innen aus Kairo beschreiben den Tahrirplatz als eine Art rechtsfreien Raum (vgl. El-Nadeem 2013: 29), eine Einschätzung, die von Amnesty International geteilt wird. Die über 500 Fälle von Gruppenvergewaltigungen auf dem Tahrirplatz von Juni 2012 bis Juni 2014 seien aufgrund fehlender Untersuchungen durch Mangel an politischem Willen nicht aufgeklärt worden (vgl. AI 2015: 10). Die Bestrafung von Frauen* für ein nicht (gender)normkonformes Verhalten im öffentlichen Raum und die Wiederherstellung von autoritären Strukturen im Zusammenspiel mit einer angenommenen Straffreiheit kann als ein Auslöser der Übergriffe angesehen werden. Problematisch an dieser Lesart der Ereignisse ist die Rückführung auf sexuelle Lust und das dadurch determinierte Verhalten männlicher* Individuen, wodurch die strukturelle Komponente sexualisierter Gewalt ausgeblendet bleibt. Dieser Rückgriff auf den Sexualtrieb entmenschlicht die Täter und spricht ihnen eine eigene Willenskraft ab, da letztlich davon ausgegangen 
wird, dass jeder Mann eine Veranlagung für sexuelle Aggression und Gewalt in sich trägt. Dies ermöglicht wiederum eine Lesart, in der Täter individuell versagt haben. Es ist deshalb wichtig, das konkrete Einzelfallgeschehen als im Auftreten oft vergleichbar zu markieren, um es als gesellschaftliches Phänomen und damit auch als gesamtgesellschaftliches Problem zu begreifen. Weitergehend trägt obige Interpretation zu Vergewaltigungsmythen bei, indem er die Täter zu Opfern ihrer eigenen biologischen Konstitution macht. Damit entmenschlicht er und negiert lebensgeschichtliche und kontextuale Einflüsse. Es scheint so als würde nur Bestrafung sexualisierte Gewalt verhindern. Auch passt diese Lesart zur von Hark und Villa (2017: 41) herausgearbeiteten Figur des ,,zu sexueller Triebkontrolle [...] nur unzureichend befähigten, inferioren muslimischen Jungmannes ohne familiäre Bindung und Kontrolle." Doch erst vor dem beschriebenen Hintergrund um hegemoniale Männlichkeit und Anerkennungskämpfen lassen sich die Übergriffe präziser einordnen.

Sexualisierte Gewalt ist somit in zweierlei Art mit der vom Staat durchgesetzten Ordnung verknüpft: Sie kann aus einer Situation erwachsen, in der angenommen wird, keine Bestrafung befürchten zu müssen. Dies war im Kairoer Beispiel gegeben, sowohl durch das Desinteresse, als auch die direkte Beteiligung der Sicherheitskräfte an sexualisierter Gewalt. Die Polizei wurde im jeweiligen Moment als nicht durchschlagskräftig eingeschätzt, war sie doch nicht in der Lage, die Demonstrationen aufzulösen. In diesem Kontext ist es möglich, die Übergriffe in Kairo als Versuch der alten Ordnung zu lesen, mit der Verdrängung von Frauen* aus der öffentlichen Sphäre das patriarchale System wiederherzustellen. Über die Wiederherstellung der Geschlechterverhältnisse werden auch politische Strukturen rekonstruiert. In Köln entstand in den Stunden der Silvesternacht um den Bahnhof herum ein rechtsfreier Raum, in dem die Möglichkeit für nicht-normkonformes Verhalten ohne Bestrafung gegeben schien, so Mounir, einer der Täter (vgl. Amjahid et al. 2016: 18). Weitergehend können patriarchale Geschlechterverhältnisse auch als systemkonstituierend eingeschätzt werden. 


\section{SEXUALISIERTE GEWALT ALS MITTEL DER KONSTRUKTION DES EIGENEN UND FREMDEN}

Farha Ghannam (2002: 100 Hervorhebung im Original) zeigt im Kontext sexualisierter Belästigung mit Bezug auf ägyptische Männer* eine klare Aufteilung zwischen fremden und eigenen Frauen*, also jenen, die zur eigenen Gruppe und zum sozialem Umfeld gehören, welches über Raumzuweisungen hergestellt wird:

„While teasing and making comments that target young women from outside their immediate surroundings are perceived as a source of enjoyment and entertainment, young men should refrain from targeting women from their murabba'at or streets.“

Das Konzept zwischen eigenen und fremden Frauen zu unterscheiden findet sich auch in Deutschland und tritt im Kölner Fall in der Unterscheidung unserer Frauen und anderer Männer zu Tage, wenn nun weiße Männer weiße Frauen vor „,braunen“ Männern schützen. Im Ministeriumsbericht äußert sich dies durch die Gegenüberstellung einer weiblichen Opfergruppe ohne Nationalität und einer männlichen Tätergruppe, bei der ununterbrochen auf ihre Herkunft abgehoben wird. Hier zeigt sich, dass es bereits ein Narrativ davon gibt, wie Opfer sexualisierter Gewalt aussehen (und weitergehend auch welcher Umgang mit dem Überleben akzeptiert und glaubhaft ist). Überlebende sind in diesem Narrativ cis-weiblich, heterosexuell, wei $\beta$ und able-bodied. ${ }^{12}$ Das sich auch im Diskurs nach der Silvesternacht Whiteness als eine unmarkierte Kategorie äußert, die gleichzeitig für die Konstruktion des Eigenen benutzt wird, zeigt, dass ein Nachdenken über dieses Narrativ notwendig ist.

12 Weiß ist nicht hier als biologische Tatsache gemeint, sondern als eine weiße Menschen privilegierende soziale Konstruktion, die sich über die Abgrenzung zu nicht-weißen anderen konstruiert. Able-bodied meint eine Bezeichnung für Körper, die gesellschaftlich als psychisch und physisch gesund gelten. Robert McRuer (2006) zeigt wie sich Zwangsheterosexualität (compulsory heterosexuality) und normativ als gesund wahrgenommene Körper (compulsory able-bodiedness) bedingen und deshalb zusammen gedacht werden müssen um die symbolisch-materiellen Institutionalisierungen von Körpernormen zu dekonstruieren. 
Da sich weite Teile der deutschen Gesellschaft noch immer nicht als Einwanderungsgesellschaft positionieren möchten, werden ethnische Zuschreibungen als natürliche Attribute angenommen, die in geographischer Herkunft, körperlichen Charakteristika und kulturellen Benehmen sowie Gruppenbildung angeblich ihren unzweideutigen Ausdruck finden. Die Täter werden dabei als nicht-deutsch verortet, also im geographischen wie kulturell nicht zugehörigen Sinne zum Anderen gemacht. Dadurch kann dann angenommen werden, dass die Übergriffe auf dem Bahnhofsvorplatz in Köln zur Abwertung und Verhöhnung deutscher Männer dienten, wie beispielsweise von Alice Schwarzer (2016) und Bassam Tibi (2016) suggeriert.

Eigenes und Fremdes muss sich dabei nicht zwangsläufig auf (angenommen) ethnische Gemeinschaften beziehen. So fokussierte sich der Diskurs bereits vor der sexualisierten Gewalt auf dem Tahrirplatz auf ,workingclass“ Männer und dämonisierte diese Gruppe als „Belästiger“, während gleichzeitig Mittelklasse-Frauen als Opfer gegenübergestellt wurden (vgl. Amar 2011: 314). Dies lenkt den Blick auf Klasse als Analysekategorie, zeigt erneut, dass sexualisierte Gewalt nicht außerhalb ihrer Kontexte verhandelt werden kann - und fügt den Ereignissen auf dem Tahrirplatz eine weitere Machtstruktur hinzu. Während es also auf dem Tahrirplatz Männlichkeit in Kombination mit Klasse war, wurde in Deutschland Migration zusammen mit Männlichkeit für eine alleinige Zuschreibung an eine bestimmte Personengruppe genutzt. Je besser diese Verallgemeinerungen in das Bedürfnis zur Aufwertung der eigenen Gruppe passt, desto besser funktioniert laut Thomas Pettigrew (1979) die Generalisierung der negativen Handlungen von Individuen auf eine Gesamtgruppe. Positive Handlungen von (so wahrgenommenen) Angehörigen dieser Gruppe werden dabei als Ausnahmen kategorisiert. Pettigrew nennt dies den ultimativen Zuschreibungsfehler.

Hierbei geht es einerseits darum eine Illusion der Mehrheitsgesellschaft aufrecht zu erhalten und den weißen westlichen Mann bzw. die ägyptische Gesellschaft als vernünftig, modern und zivilisiert zu konstruieren, während die Gewalttätigkeit bei den Anderen verortet wird. Andererseits führen die Zuschreibungen zu konkreten politischen Maßnahmen. Nach der Kölner Silvesternacht wurde die legitimierte staatliche Gewalt massiv erhöht, was direkten Einfluss auf das Leben vieler (post-)migrantischer Kölner_innen hatte (vgl. z.B. Charchira in Junghans 2016). Auch nach den Übergriffen in Kairo wurden Forderungen nach polizeilicher Intervention laut (vgl. Amar 2011), obwohl die Sicherheitskräfte auf dem Tahrirplatz während der Ereignisse 
nicht einschritten oder gar selbst beteiligt waren, beziehungsweise die Autoritäten Überlebende nach ihren Erlebnissen auf erniedrigende Weise behandelten (vgl. AI 2015; El-Nadeem 2013: 26f; 34f). In Deutschland kann dieser Ruf wiederum in den Kontext illegitimer Gewalt gestellt werden, waren doch zum Zeitpunkt der Übergriffe nur die wenigsten dieser Taten strafbar, da das „deutsche Strafrecht [...] wenig bis gar keinen Schutz gegen sexuelle Belästigung im öffentlichen Raum“ (Lembke 2016) bot. Dies wurde bereits vor den Kölner Ereignissen von feministischer Seite kritisiert. Die Forderung nach einer härteren Bestrafung berührt Fragen der Rechtssicherheit und ist damit illegitim. Die Kölner Silvesternacht hatte direkten Einfluss auf die Veröffentlichung des Gesetzentwurfs zur Einführung beschleunigter Asylverfahren (Asylpaket II) im Februar 2016 (vgl. Deutscher Bundestag 2016). Auch die Einstufung von Algerien, Tunesien und Marokko als sichere Herkunftsländer im Mai 2016 durch den Bundestag, welche später vom Bundesrat abgelehnt wurde, sowie die Vorwürfe des racial profiling gegen die Kölner Polizei in der Silvesternacht 2016, können in direkten Zusammenhang mit der Verhärtung des Diskurses um Migration nach der Kölner Silvesternacht gesehen werden. Inwieweit die obigen Zuschreibungen sich auch in den Nationalitäten der angeklagten Täter äußern, sie also bei Beschreibungen durch Polizei und Betroffene eine Rolle gespielt haben mögen, kann hier nicht eruiert werden. In Köln zeigte sich im Bericht einer Zeugin, dass sie von den ermittelnden Beamt_innen bereits in der Nacht mehrmals die Frage: „Das waren doch Flüchtlinge?“ gestellt bekommen hatte, obwohl sie darauf bestand, die Herkunft der Täter nicht zu kennen (vgl. Amjahid et al. 2016: 21). Die Beamt_innen benutzten bei der Beschreibung der Täter die Termini „,arabische Männer“, mit „,südländischem“ bzw. „,nordafrikanischem Aussehen“, „Menschen mit Migrationshintergrund“ und „Ausländer“ (MIK 2016). Diese Bezeichnungen dienen dabei nicht nur der Klärung des Wir und Ihr, mit der Verwendung einer räumlichen Bezeichnung werden die Subjekte auch sozial verortet. Auch ist kritisch zu hinterfragen, welche Ermittlungspraktiken schließlich zu den bisher festgestellten 183 Angeklagten geführt haben, wenn vor dem Hauptbahnhof bis zu 1000 Menschen anwesend waren. ${ }^{13}$ So beschrieb eine Richterin im Bezug auf die Aufarbeitung der Übergriffe in Hamburg erhebliche Ermittlungsfehler:

13 In Deutschland ist racial profiling zwar verboten, jedoch führt die Bundespolizei „im Rahmen von $\S 22$ Abs. 1 a BPolG diskriminierende Personenkontrollen“ 
„Sie habe den Eindruck, dass die Ermittler Ergebnisse um jeden Preis präsentieren wollten - notfalls , auf Kosten rechtsstaatlicher Grundsätze‘. Sie sei ,erschüttert und erschrocken über das, was das Verfahren zutage gebracht hat' . Die Ermittlungsarbeit der Polizei, so die Richterin, war ,keine Überführung, sondern schlicht und ergreifend: raten'، (Kempkens 2016)

Aber nicht nur der Staat reagierte mit Gewalt. Der starke Anstieg der Straftaten gegen Flüchtlingsunterkünfte kann vor dem Hintergrund der „latenten Radikalisierung des bereits bestehenden gesellschaftlichen Diskurses“ (BKA 2016: 7f) und den sich dezidiert nach Köln gebildeten Bürgerwehren als eine Reaktion durch nicht legitimierte Gewaltformen begriffen werden. ${ }^{14}$ In Köln und Düsseldorf wurden im Januar 2016 Bürgerwehren gegründet, die zumindest in Köln gewalttätig gegenüber nicht-weißen Personen wurden (vgl. Jacobsen 2016). Diese Bürgerwehren waren eine Reaktion auf die Kölner Silvesternacht. So schreibt die Kölner Bürgerwehr (2016) auf Facebook: ,„[..] wir werden gegen diese Leute[,] die unseren [sic!] Töchtern [sic!] am vergangenen Sylvester [sic!] bedrängt haben[,] vorgehen. “ Wehren können sich Personen erst, wenn sie angegriffen wurden. Die Formierung einer Bürgerwehr außerhalb eines konkreten Bedrohungsmoments dient damit nicht zum Selbstschutz, sondern der Verstärkung bereits bestehender Abwehrmechanismen. Sie bedient zudem (hetero)sexistische Ideen von schutzbedürftigen Frauen und verstärkt rassistische Ressentiments.

durch, die als ,grund- und menschenrechtswidrige[s] Verhalten“ bezeichnet werden können. Das Gesetz ist darauf ausgelegt, dass Bundespolizist_innen ,,anhand von Pauschalverdächtigungen selektive und damit rassistische Personenkontrollen vornehmen“ (Cremer 2013: 32). Durch einen solchen Gesetzesauftrag werden - abgesehen von den mit der diskriminierenden Praxis einhergehenden Rechtsverletzungen im Einzelfall - ,,rassistische Stereotype in der Bevölkerung wie auch in der Polizei selbst verstärkt" (ebd.).

14 Für die Beschreibung der Bürgerwehren wurde hier das generische Maskulinum benutzt. Frauen* sind mitunter Teil dieser Gruppen, allerdings ist ihre Zahl vergleichsweise gering. Auch werden in der Kommunikation der Bürgerwehren vornehmlich männlich attribuierte Eigenschaften betont. 


\section{SEXUALISIERTE GEWALT ALS DOING SPACE ZUR (RE-)PRODUKTION VON MÄNNLICHKEIT}

Die Gleichsetzung der sexualisierten Gewalt auf dem Kairoer Tahrirplatz und dem Bahnhofsvorplatz ist, wie gezeigt wurde, nicht nur vor dem Hintergrund postkolonialer Theorie unzulässig, sie vernachlässigt durch den Rückbezug auf ein einziges Merkmal des ,arabischen/nordafrikanischen“ Mannes andere wichtige Einflussfaktoren. [TRANSSEKTIONALITÄT] Die Gegenüberstellung beider Fallbeispiele hat gezeigt, dass sexualisierte Gewalt nicht außerhalb ihrer lokalen Kontexte verhandelt werden kann und sollte. Trotzdem darf der globalhistorische und globalisierte Zusammenhang nicht komplett außer Acht gelassen werden, verbinden sich Kairo und Köln doch auch in der Frage, welche Partizipationsmöglichkeiten Menschen unter den momentanen sozioökonomischen Umbrüchen haben. In Kairo wurde die Teilhabe an der Ausübung bürger_innenlicher Rechte und politischer Entscheidungen durch den Übergriff auf Frauen*körper im öffentlichen Raum verhandelt. Die Übergriffe in der Kölner Silvesternacht können hingegen nach Ergebnis dieser Analyse eher als Ringen um gesellschaftliche Teilhabe und das eigene Gesehen-werden, sowie die Kompensation von Marginalisierung interpretiert werden. Mit Hilfe der Aneignungsperspektive wurde das abweichende Verhalten der jungen Männer* als Raumhandeln aufgeschlüsselt, wodurch die Übergriffe auf Frauen*körper als ein Mittel zur Herstellung von situativer oder temporärer Herrschaft sichtbar wurden. Dabei spielen die Performanz in öffentlichen Räumen wie auch der Zugang zu ebenjenen eine entscheidende Rolle, denn im Raum werden Machtverhältnisse verhandelt. Das Aneignen des Raumes in Köln und Kairo wird dadurch gleichzeitig auch zum doing gender, die Reproduktion patriarchaler Geschlechterverhältnisse über räumliche und körperliche Aneignung dient der Aufrechterhaltung und dem Zugewinn von Macht. Das Aneignen des Raumes, als doing space gelesen, ist dann Teil der Herstellung von Geschlecht. Die Bezugnahme auf das doing gender als Grundlage für männliches Handeln in Abgrenzung vom und Erhöhung über das andere Weibliche*, scheint auch hier sinnvoll. Zur alleinigen Erklärung ist es allerdings ebenfalls unzureichend, da ein Rückgriff auf eine Allgemeingültigkeit beanspruchende Kausalannahme in Gefahr essentialisierender Erklärungsmuster läuft. Statt Gewalt linear auf eine einzelne Ursache zurückzuführen, sollten Untersuchungen und Diskussionen der Gründe für Gewalt den Blick für die Vielzahl von Faktoren offenhalten, die 
in den Lebensrealitäten der tatbegehenden Personen eine Rolle spielen. Selbst die Erweiterung der Faktoren von Herkunft und Männlichkeit hin zu einer differenzierteren Untersuchung von MännlichkeitEN und Marginalisierung bietet allenfalls neue Interpretationsangebote, wobei die Untersuchung weiterer Faktoren, wie der Konsum berauschender Mittel und die Tatbegehungsform aus der Gruppe heraus, wie an anderer Stelle vorgenommen, noch weitere Differenzierungen zulassen (vgl. Schmoliner 2017).

Im vorliegenden Quellenmaterial aus Köln und Kairo wurde die Kontrastierung der binären Strukturen der Opferfrau und des Tätermanns festgestellt, wodurch beide Geschlechter unzulässig reduziert werden. Einer Annahme von Männlichkeit als generell machtvoll lässt sich mit Connells Konzept der hegemonialen Männlichkeit widersprechen und die Raumaneignung über den Frauen*körper als das Ausüben protestierender Männlichkeit im Kölner Fall aufzeigen, während in Kairo der Versuch offenbar wird, über die Wiederherstellung der Geschlechterordnung auch die gesellschaftliche Ordnung wiederherzustellen. Lässt sich die für die Konstruktion von Männlichkeit nötige Macht nicht mehr auf andere Art herstellen, bzw. ist die patriarchale Gesellschaftsordnung durch die Performanz von Frauen* im öffentlichen Raum im Begriff einer Transformation, greifen Männer auf Gewalt als letzte Ressource zurück. Ohne diese Dimension der Geschlechterordnung kann männliche Gewalt nur ungenügend eingeordnet werden, da in den binären Strukturen zwischen männlichen Tätern und weiblichen Opfern verblieben wird, ohne die Zwischenräume in Betracht zu ziehen. Die Betrachtung der Zwischenräume ermöglicht es dem auch durch die Quellen fortgeschriebenen Narrativ, Überlebende seien ausschließlich cis-weiblich, heterosexuell, weiß und able-bodied und zudem passive Opfer, entgegenzuwirken und die Handlungsmacht von Frauen* anzuerkennen.

Ein Großteil des Diskurses in der deutschen Öffentlichkeit zeichnete derweil die Männlichkeit der jugendlichen Täter als abnormal gegenüber der Norm deutscher Männer*. Hierdurch ergibt sich eine Kennzeichnung des Anderen als wild und rückständig, während das Eigene als zivilisiert dargestellt wird. So werden also das Eigene und das Andere auch über die Binarität gewaltlos und gewalttätig konstruiert. Gewalt wird in Distanz und Devianz verortet. Das bedeutet, gewalttätig ist dann vor allem der Globale Süden, aber auch das in Kairo durch Klassengrenzen abgesteckte „Konfliktmilieu“. Hier sollte hervorgehoben werden, dass okzidentale Selbstinszenierungen über di- 
chotomisierende Ab- und Ausgrenzungen kein neues Phänomen sind, sondern koloniale Diskurse weiterführen. Die Konstruktion problematischer anderer Männer ist nicht neu, sondern greift auf vorhandenes (koloniales) Wissen und darüber legitimierte Herrschaftspraktiken zurück und reproduziert diese unter veränderten sozialen Bedingungen. Die Motive des Schutzes der eigenen Frauen vor den anderen Männern wiederholen sich in verschiedenen Kontexten und auf verschiedene Personengruppen bezogen. Die post-KölnDiskurse führen die vorher benutzten rassistischen wie sexistischen Diskursmuster weiter. Vor dem Hintergrund der Kolonisation wie auch den noch immer vorherrschenden globalen Ungleichheiten ist die in Deutschland vorgenommene Zuschreibung gravierend, hat sie doch, wie gezeigt, direkte Auswirkungen auf politische Entscheidungen gehabt. Die Frage nach dem Umgang mit den Ereignissen der Kölner Silvesternacht berührt demnach nicht nur Identitätskonflikte der Anderen, sondern beleuchtet auch die Verfasstheit der deutschen Gesellschaft. Die Bildung von Bürgerwehren und das racial profiling der Polizei an Silvester 2016 in Köln lassen dabei auf eine Reaktion auf illegitime Gewalt mit illegitimer Gewalt schließen, die im Nachhinein normalisiert und verteidigt wurde.

\section{FAZIT}

Auch wenn die Beziehungen und Überschneidungen zwischen race, Klasse und Geschlecht hier aufgezeigt wurden und offenbar wird, dass ein alleiniges Abstellen auf die Herkunft der Täter zu einer einseitigen Deutung der Ereignisse führt, lassen sich die vorliegenden Ergebnisse nicht als eine „Rache der Unterdrückten“ interpretieren. Ebenso wie für das Merkmal Migration ist auch eine Verengung auf Marginalisierung unzulässig, führt sie doch zu einer Viktimisierung der Täter. Die vielfältigen Einflussfaktoren, die durch die Einordnung der Ereignisse an beiden Schauplätzen als Verhandlungsprozesse von Machtverhältnissen offenbar werden, zeigen, dass der Vergleich selbst ein Akteur im Spannungsfeld postkolonialer Machtverhältnisse ist, in welchem der Unterschied zwischen ,,arabisch/nordafrikanischen“ Männern und ,zivilisiert-modernen Deutschen“ konstruiert wird. Von einem „TahrirPlatz in Köln“ zu sprechen, wie es Alice Schwarzer und andere tun bleibt unzulässig und ist möglicherweise vielmehr Teil eines gewaltsamen Kon- 
flikts als Beitrag zu dessen Lösung. Die Differenzen zwischen beiden Ereignissen wurden jedenfalls hier herausgearbeitet. Hervorzuheben ist, dass in beiden Fällen Frauen*körper zur Verhandlungsfläche um gesellschaftliche und politische Macht wurden. Die Analyse zeigt, dass Männlichkeit im Zusammenhang mit Migration in intersektionaler Perspektive auf gegenwärtige Grenzziehungen und Selbstvergewisserungen eine wichtige Markierungsfunktion zukommt. Gleichzeitig wurde offenbar, dass geschlechtsspezifische Prozesse im Zusammenspiel mit den desintegrierenden Folgen sozialer Entwicklungen und in Ergänzung situativer Anlässe ein aggressives Gewalthandeln nach sich ziehen können. Konfigurationen von hegemonialer Männlichkeit sind darin ein entscheidender Hauptakteur.

\section{LITERATUR}

Abdelmonem, Angie (2016): Anti-Sexual Harassment Activism in Egypt. Transnationalism and the Cultural Politics of Community Mobilization. Unveröffentlichte Dissertation. Arizona State University.

Abu-Khalid, Fowziyah A. (2003): „Interview on Current Challenges Facing the Arab Women's Movements“, in: Al-Raida XX 100, S. 50-53.

Aikins, Joshua Kwesi/Bendix, Daniel (2010): „PERIPHERIE-Stichwort: post(-)kolonial“", in: Peripherie 120, S. 478-481.

Amar, Paul (2011): ,Turning the Gendered Politics of the Security State Inside Out?", in: International Feminist Journal of Politics 13, S. 299-328.

Amjahid, Mohamed/Fuchs, Christian/Guinan-Bank, Vanessa/Kunze, Anne et al. (2016): „Was geschah wirklich?“, in: ZEITmagazin 27, S. 16-34.

Amnesty International (2015): ,Circles of Hell': Domestic, Public and State Violence Against Women in Egypt, London: AI.

Badran, Margot (1995): Feminists, Islam, and Nation. Gender and the Making of Modern Egypt, Princeton: Princeton University Press.

Bauriedl, Sybille/Schier, Michaela/Strüver, Anke (2010): „Räume sind nicht geschlechtsneutral. Perspektiven der geographischen Geschlechterforschung“, in: Sybille Bauriedl/Anke Strüver (Hg.), Geschlechterverhältnisse, Raumstrukturen, Ortsbeziehungen. Erkundungen von Vielfalt und Differenz im ,spatial turn', Münster: Westfälisches Dampfboot, S. 1025. 
Bier, Laura (2011): Revolutionary Womanhood. Feminisms, Modernity, and the State in Nasser's Egypt. Stanford: Stanford University Press.

Bourdieu, Pierre (1991): „Physischer, sozialer und angeeigneter physischer Raum“, in: Martin Wentz (Hg.): Stadt-Räume, Frankfurt a. M./New York: Campus Verlag, S. 25-34.

Bourdieu, Pierre (2005): Die männliche Herrschaft. Frankfurt a. M.: Suhrkamp.

Braun, Christina von/Mathes, Bettina (2007): Verschleierte Wirklichkeit.

Die Frau, der Islam und der Westen, Berlin: Aufbauverlag.

Bründel, Heidrun/Hurrelmann, Klaus (1994): Gewalt macht Schule, München: Droemer Knaur.

Bundeskriminalamt (2016): Kriminalität im Kontext von Zuwanderung Kernaussagen 1/2016, Wiesbaden: Bundeskriminalamt.

Butler, Judith (1988): „Performative Acts and Gender Constitution: An Essay in Phenomenology and Feminist Theory“, in: Theatre Journal 40, S. 519-531.

Butler, Judith (1997): Körper von Gewicht. Frankfurt a. M.: Suhrkamp.

Butler, Judith (2016): Anmerkungen zu einer performativen Theorie der Versammlung. Berlin: Suhrkamp.

Castro Varela, María do Mar/Dhawan, Nikita (2005): Postkoloniale Theorie. Eine kritische Einführung, Bielefeld: transcript.

Connell, Raewyn ([1987] 2015): Der gemachte Mann. Konstruktion und Krise von Männlichkeiten, Wiesbaden: Springer VS.

Cremer, Hendrik (2013): ,Racial Profiling‘. Menschenrechtswidrige Personenkontrollen nach $\S 22$ Abs. 1a Bundespolizeigesetz; Empfehlungen an den Gesetzgeber, Gericht und Polizei, Berlin: Dt. Institut für Menschenrechte.

Deutscher Bundestag (2016): Debatte vom 19. Februar 2016.

Drüeke, Ricarda (2016): Die TV Berichterstattung in ARD und ZDF über die Silvesternacht 2015/16 in Köln. E-Paper der Heinrich-Böll-Stiftung.

El-Feki, Shereen/Heilman, Brian/Barker, Gary (Hg.) (2017): Understanding Masculinities, Kairo/Washington DC: UN Women/Promundo.

El-Nadeem Center for Rehabilitation of Victims of Violence and Torture (2013): Sexual Assault and Rape in Tahrir Square and its Vicinity: A Compendium of Sources 2011-2013, Kairo: Nazra for Feminist Studies. 
Engels, Bettina (2015): „Zwischen Mainstream und Kritik: Postkoloniale Perspektiven in der Friedens- und Konfliktforschung“, in: Matthias Bös/Lars Schmitt/Kerstin Zimmer (Hg.), Konflikte vermitteln? Lehren und Lernen in der Friedens- und Konfliktforschung, Wiesbaden: Springer Fachmedien, S. 305-322.

Filiu, Jean-Pierre (2011): The Arab Revolution. Ten Lessons from the Democratic Uprising, Oxford/New York: Oxford University Press.

Ghannam, Farha (2002): Remaking the Modern. Space, Relocation, and the Politics of Identity in a Global Cairo, Berkeley: University of California Press.

Giddens, Anthony (1988): Die Konstitution der Gesellschaft, Frankfurt a. M.: Campus-Verlag.

Gregory, Derek (2013): „Tahrir. Politics, Publics and Performances of Space“, in: Middle East Critique 22, S. 235-246.

Hafez, Sherine (2012): „No Longer a Bargain. Women, Masculinity, and the Egyptian Uprising“, in: American Ethnologist 39, S.37-42.

Hafez, Sherine (2014): „The Revolution Shall not Pass through Women's Bodies. Egypt, Uprising and Gender Politics“, in: The Journal of North African Studies 19, S. 172-185.

Hagemann-White, Carol (1984): Sozialisation: Weiblich-männlich?, Opladen: Leske + Budrich

Harders, Cilja (2009): „Politik von unten - Perspektiven auf den autoritären Staat in Ägypten“, in: Martin Beck/Cilja Harders/Annette Jünemann (Hg.): Der Nahe Osten im Umbruch. Zwischen Transformation und Autoritarismus. Wiesbaden: VS Verlag, S. 299-323.

Hark, Sabine/Villa, Paula-Irene (2017): Unterscheiden und herrschen. Ein Essay zu den ambivalenten Verflechtungen von Rassismus, Sexismus und Feminismus in der Gegenwart, Bielefeld: transcript.

Harvey, David (1989): The Condition of Postmodernity, Oxford/Cambridge: Basil Blackwell.

Hatem, Mervat F. (1992): Economic and Political Liberation in Egypt and the Demise of State Feminism, in: International Journal of Middle East Studies 24, S. 231-251.

Jacobsen, Lenz (2016): „Bürgerwehr: Sicherheit, selbst gemacht“, in: ZEIT online vom 15.01.2016. 
Joseph, Suad (1997): „The Public/Private: The Imagined Boundary in the Imagined Nation/State/Community: The Lebanese Case“, in: Feminist Review 57, S. 73-92.

Junghans, Daniela (2016): „Teil 2: Die politischen Folgen“, in: WDRMorgenecho vom 20.12.2016.

Kandiyoti, Deniz (1988): „Bargaining with Patriarchy“, in: Gender and Society 2, S. 274-290.

Kempkens, Sebastian (2016): „Sexuelle Übergriffe: Richterin wirft Ermittlern zur Silvesternacht grobe Fehler vor", in: ZEIT online vom 01.11.2016.

Kimmel, Michael (2007): „Contextualizing Men’s Violence. The Personal Meets the Political“, in: Laura O’Toole/Jessica Schiffman/Margie L. Kiter (Hg.): Gender Violence. Interdisciplinary Perspectives, New York: New York University Press, S. 99-110.

Klaus, Elisabeth/Drüeke, Ricarda (2010): „Öffentlichkeit und Privatheit: Frauenöffentlichkeiten und feministische Öffentlichkeiten“, in: Ruth Becker/Beate Kortendiek/Barbara Budrich (Hg.), Handbuch Frauen- und Geschlechterforschung. Theorie, Methoden, Empirie, Wiesbaden: VS Verlag, S. 244-251.

Kölner Bürgerwehr (2016): Facebook-Eintrag vom 6. Januar 2016.

Koning, Anouk de (2009): Global Dreams. Class, Gender, and Public Space in Cosmopolitan Cairo, London: I.B. Tauris.

Krasmann, Susanne/Martschukat, Jürgen (2007): ,Rationalitäten der Gewalt. Staatliche Neuordnungen vom 19. bis zum 21. Jahrhundert - eine Einführung“, in: dies. (Hg.), Rationalitäten der Gewalt. Staatliche Neuordnungen vom 19. bis zum 21. Jahrhundert, Bielefeld: transcript, S. 7-18.

Kutschinske, Karin/Meier, Verena (2000): „,... sich diesen Raum zu nehmen und sich freizulaufen...'. Angst-Räume als Ausdruck von Geschlechterkonstruktion“, in: Geographica Helvetica 55, S. 138-145.

Lembke, Ulrike (2016): Sexuelle Übergriffe im öffentlichen Raum - Rechtslage und Reformbedarf in Deutschland. Text abrufbar unter: http://www.legal-gender-studies.de/sexuelle-uebergriffe-imoeffentli chen-raum-rechtslage-und-reformbedarf\#_edn18, besucht am 10. 1. 2017.

Löw, Martina/Steets, Silke/Stoetzer, Sergej (2007): Einführung in die Stadtund Raumsoziologie, Opladen u.a.: Budrich-Verlag. 
Marx Ferree, Myra/Tripp, Aili M. (Hg.) (2006): Global Feminism. Transnational Women's Activism, Organizing, and Human Rights, New York/London: New York University Press.

McLarney, Ellen Anne (2015): Soft Force. Women in Egypt's Islamic Awakening, Princeton: Princeton University Press.

McRuer, Robert (2006): „Compulsory Able-Bodiedness and Queer/Disabled Existence“, in: Davis, Lennard J. (Hg.), The Disability Studies Reader, London/New York: Routledge, S. 88-99.

Messerschmidt, James (1993): Masculinities and Crime. Critique and Reconceptualization of Theory, Lanham, Md.: Rowman \& Littlefield.

Meuser, Michael (2002): „,Doing Masculinity“. Zur Geschlechtslogik männlichen Gewalthandelns", in: Regina-Maria Dackweiler/Reinhild Schäfer (Hg.), Gewalt-Verhältnisse. Feministische Perspektiven auf Geschlecht und Gewalt. Frankfurt a. M.: Campus-Verlag, S. 53-78.

Ministerium für Inneres und Kommunales des Landes Nordrhein-Westfalen (MIK) (2016): Bericht des Ministeriums für Inneres und Kommunales über die Übergriffe am Hauptbahnhof Köln in der Silvesternacht, Düsseldorf.

Mohanty, Chandra T. (1984): „Under Western Eyes: Feminist Scholarship and Colonial Discourses“, in: Boundary 2, 12, S. 333-358.

Mohanty, Chandra T. (2003): Feminism without Borders. Decolonizing Theory, Practicing Solidarity, Durham NC: Duke University Press.

Pettigrew, Thomas F. (1979): „The Ultimate Attribution Error: Extending Allport's Cognitive Analysis of Prejudice“, in: Personality and Social Psychology Bulletin 5, S. 461-476.

Reuter, Julia/Villa, Paula-Irene (2010): „Provincializing Soziologie. Postkoloniale Theorie als Herausforderung", in: Julia Reuter/Paula-Irene Villa (Hg.): Postkoloniale Soziologie. Empirische Befunde, theoretische Anschlüsse, politische Intervention, Bielefeld: transcript, S. 7-46.

Ruhne, Renate (2003): Raum, Macht, Geschlecht. Zur Soziologie eines Wirkungsgefüges am Beispiel von (Un)Sicherheiten im öffentlichen Raum, Opladen: Leske + Budrich.

Salama, Hussam H. (2013): „Tahrir Square: A Narrative of a Public Space“, in: International Journal of Architectural Research 7, S. 128-138.

Schmincke, Imke (2009): Gefährliche Körper an gefährlichen Orten, Bielefeld: transcript. 
Schmoliner, Marei (2017): ,Tahrir-Platz in Köln“? - Zur Aneignung des öffentlichen Raumes durch sexualisierte Gewalt als Mittel der (Re-)Produktion von Männlichkeit. Unveröffentlichte Masterarbeit, Erfurt.

Schneider, Silke/Wilde, Gabriele (2012): „Falsche Sicherheiten. Geschlechterverhältnisse in autoritären Regimen“, in: Femina Politica 21, S. 9-16.

Schroer, Markus (2007): „Defizitäre Reziprozität: Der Raum der Überflüssigen und ihr Kampf um Aufmerksamkeit“, in: Cornelia Klinger/GudrunAxeli Knapp/Birgit Sauer (Hg.), Achsen der Ungleichheit. Zum Verhältnis von Klasse, Geschlecht und Ethnizität, Frankfurt a. M.: Campus Verlag, S. 257-270.

Schwarzer, Alice (2016): „Silvester 2015, Tahrir-Platz in Köln“, in: Alice Schwarzer (Hg.), Der Schock - die Silvesternacht in Köln, Köln: Kiepenheuer \& Witsch, S. 7-39.

Silverstein, Paul (2008): „,Thin Lines on the Pavement. The Racialization and Spatialization of Violence in Postcolonial (Sub)Urban France“, in: Martina Rieker/Kamran Asdar Ali (Hg.), Gendering Urban Space in the Middle East, South Asia, and Africa, New York: Palgrave Macmillan, S. 169205.

Sokoloff, Natalie J./Dupont, Ida (2005): ,Domestic Violence at the Intersections of Race, Class, and Gender: Challenges and Contributions to Understanding Violence against marginalized Women in diverse Communities“, in: Violence Against Women 11, S. 38-64.

Sorbera, Lucia (2014): „Challenges of Thinking Feminism and Revolution in Egypt between 2011 and 2014“, in: Postcolonial Studies 17, S. 63-75. Spitthöver, Maria (1990): „Frauen und Freiraum“, in: Kerstin Dörhöfer (Hg.): Stadt-Land-Frau: soziologische Analysen; feministische Planungsansätze, Freiburg: Kore, S. 81-103.

Spivak, Gayatri C. (1988): „Can the Subaltern Speak?“, in: Cary Nelson /Lawrence Grossberg (Hg.), Marxism and the Interpretation of Culture, Chicago: University of Illinois Press, S. 271-314.

Strüver, Anke (2003): „,Das duale System“: Wer bin ich - und wenn ja, wie viele? Identitätskonstruktionen aus feministisch poststrukturalistischer Perspektive“, in: Hans Gebhardt/Harald Bathelt (Hg.), Kulturgeographie. Aktuelle Ansätze und Entwicklungen, Heidelberg: Spektrum-Verlag, S. 113-128. 
Thompson, Christiane (2015): „Anerkennung und Macht in ihrer Bedeutung für die Entstehung von Gewalt", in: Wolfang Melzer/Dieter Hermann/Uwe Sandfuchs/Mechthilt Schäfer/et al. (Hg.), Handbuch Aggression, Gewalt und Kriminalität bei Kindern und Jugendlichen, Bad Heilbrunn: Julius Klinkhardt, S. 55-58.

Tibi, Bassam (2016): „Frauenrechte: ,Junge Männer, die die Kultur der Gewalt mitbringen“", in: Welt online vom 08.05.2016.

Tucker, Judith E. (1985): Women in Nineteenth - Century Egypt. Cambridge/New York: Cambridge University Press.

Wahba, Dina (2012): After the Spring II. Gender and Sexuality in Revolutionary Politics, London: SOAS.

Wood, Elizabeth (2006): „Variation in Sexual Violence during War“, in: Politics \& Society 34, S. 307-342.

Yeğenoğlu, Meyda (1998): Colonial Fantasies: Towards a Feminist Reading of Orientalism, Cambridge, U.K.: Cambridge University Press.

ZEIT online (2016): „Henriette Reker: ,Vielleicht habe ich den Frauen zu wenig Trost gespendet““, in: ZEIT online vom 22.06.2016.

Zillinger, Martin (2016): „Nafri“ als Symbol für die Flüchtlingskrise? Marokkanische Perspektiven auf Migration, in: Aus Politik- und Zeitgeschichte 33-34, S. 47-54. 